\title{
Groups having complete bipartite divisor graphs for their conjugacy class sizes
}

\author{
Roghayeh Hafezieh (*) - Pablo Spiga $(* *)$
}

ABSTRACT - Given a finite group $G$, the bipartite divisor graph for its conjugacy class sizes is the bipartite graph with bipartition consisting of the set of conjugacy class sizes of $G \backslash \mathbf{Z}(G)$ (where $\mathbf{Z}(G)$ denotes the centre of $G$ ) and the set of prime numbers that divide these conjugacy class sizes, and with $\{p, n\}$ being an edge if $\operatorname{gcd}(p, n) \neq 1$.

In this paper we construct infinitely many groups whose bipartite divisor graph for their conjugacy class sizes is the complete bipartite graph $K_{2,5}$, giving a solution to a question of Taeri [15].

Mathematics Subject Classification (2010). Primary 00Z99; secondary 99A00.

KEYWORDs. Bipartite divisor graph, conjugacy class size, extra-special group.

\section{Introduction}

Given a finite group $G$, there are several sets of invariants that convey nontrivial information about the structure of $G$. Some classical examples include the set consisting of the orders of the elements of $G$, or the set of conjugacy class sizes of $G$, or the set of degrees of the irreducible complex characters of $G$.

For every set $X$ as above, it is natural to ask to what extent the group structure of $G$ is reflected and influenced by $X$, and a useful tool in this kind of investigation is the so-called bipartite divisor graph $B(X)$ of $X$. The

(*) Indirizzo dell'A.: Department of Mathematics, Gebze Institute of Technology, Gebze, Turkey.

E-mail: roghayeh@gyte.edu.tr

(**) Indirizzo dell'A.: Dipartimento di Matematica e Applicazioni, University of Milano-Bicocca, Via Cozzi 55 Milano, MI 20125, Italy.

E-mail: pablo.spiga@unimib.it 
bipartition of the vertex set of $B(X)$ consists of $X \backslash\{1\}$ and of the set of prime numbers dividing $x$, for some $x \in X$, and the edge set of $B(X)$ consists of the pairs $\{p, x\}$ with $\operatorname{gcd}(p, x) \neq 1$.

In this paper we are interested in the case that $X$ consists of the conjugacy class sizes of $G$, that is, $X=\left\{\left|g^{G}\right| \mid g \in G\right\}$, and hence we study the bipartite divisor graph for the conjugacy class sizes. We will denote this graph simply by $B(G)$. We refer the reader to the beautiful survey [5] for the influence of the conjugacy class sizes on the structure of finite groups.

Historically, various graphs associated with the algebraic structure of a finite group have been extensively studied by a large number of authors, see for example [1, 2, 3, 4, 6, 9, 13, 16]. Recently, Lewis [14] discussed many remarkable connections among these graphs by analysing analogous of these graphs for arbitrary positive integer subsets. Then, inspired by the survey of Lewis, Praeger and Iranmanesh [12] introduced the bipartite divisor graph $B(X)$ for a finite set $X$ of positive integers and studied some basic invariants of this graph (such as the diameter, girth, number of connected components and clique number).

One of the main questions that naturally arises in this area is classifying the groups whose bipartite divisor graphs have special graphical shapes. For instance, in [10], the first author of this paper and Iranmanesh have classified the groups whose bipartite divisor graphs are paths. Similarly, Taeri [15] considered the case that the bipartite divisor graph is a cycle, and in the course of his investigation posed the following question:

Question ([15, Question 1]). Is there any finite group $G$ such that $B(G)$ is isomorphic to a complete bipartite graph $K_{m, n}$, for some positive integers $m, n \geq 2$ ?

The main theorem of this paper gives a family of examples which shows that the answer to this question is positive.

THEOREM 1.1. For every odd prime $p$, there exists a $\{2, p\}$-group $G$ with $B(G)=K_{2,5}$.

In light of Theorem 1.1, we pose the following question.

QUESTION 1.2. For which positive integers $m, n \geq 2$, is there a finite group $G$ with $B(G)=K_{m, n}$ ?

In this paper we have tried to produce infinitely many groups $G$ with $B(G)=K_{m, n}, m, n \geq 2$ and with $m, n$ as small as possible. We were unable 
to construct groups $G$ with $B(G)=K_{2,3}$. Observe that Dolfi and Jabara [8] have classified the finite groups having only two non-trivial conjugacy class sizes, and from this remarkable work it follows [8, Corollary $\mathrm{C}]$ that there is no group $G$ with $B(G)=K_{2,2}$.

REMARK 1.3. Shortly after the submission of our arXiv preprint [11] and during the refereeing process of an earlier draft of this paper, Casolo has provided us of a conjecture addressing Question 1.2. For the sake of completeness we include it here:

CONJECTURE 1.4 [7]. If $G$ is a finite group with $B(G)=K_{m, n}$ (where $n$ is the number of non-identity conjugacy class sizes of $G$ ), then $n \geq 2^{m}$.

In particular, Casolo conjectures that there exists no finite group $G$ with $B(G)=K_{2,3}$. (Casolo has proved [7] that there are groups $G$ with $B(G)=K_{2,4}$, we include a sketch of his construction in Section 3.).

Clearly a "working" conjecture can be very useful for the developing of new research, and hence we acknowledge our deepest gratitude to our friend Carlo Casolo for his contribution.

\section{Groups with complete bipartite divisor graphs for their conjugacy class sizes}

Given a finite group $G$, we denote by $\mathbf{Z}(G)$ the centre of $G$, and given $g \in G$, we denote by $g^{G}$ the conjugacy class of $g$ under $G$. Furthermore, $\left|g^{G}\right|$ denotes the cardinality of $g^{G}$.

Notation 2.1. We let $p$ be an odd prime number and $E$ be the extraspecial 2-group of plus-type

$$
\begin{array}{cc}
E=\left\langle x_{1}, \ldots, x_{p-1}, y_{1}, \ldots, y_{p-1}, z\right| & x_{i}^{2}=y_{i}^{2}=z^{2}=\left[x_{i}, z\right]=\left[y_{i}, z\right]=1 \forall i, \\
& {\left[x_{i}, x_{j}\right]=\left[y_{i}, y_{j}\right]=\left[x_{i}, y_{j}\right]=1 \forall i \neq j,} \\
& \left.\left[x_{i}, y_{i}\right]=z \forall i\right\rangle .
\end{array}
$$

Observe that

$$
\left[x_{1}^{\varepsilon_{1}} \cdots x_{p-1}^{\varepsilon_{p-1}}, y_{1}^{\eta_{1}} \cdots y_{p-1}^{\eta_{p-1}}\right]=z^{\varepsilon_{1} \eta_{1}+\cdots+\varepsilon_{p-1} \eta_{p-1}},
$$

and that every element $g \in E$ can be written uniquely as

$$
g=x_{1}^{\varepsilon_{1}} \cdots x_{p-1}^{\varepsilon_{p-1}} y_{1}^{\eta_{1}} \cdots y_{p-1}^{\eta_{p-1}} z^{v}
$$


with $\varepsilon_{i}, \eta_{i} \in\{0,1\}$ for each $i \in\{1, \ldots, p-1\}$ and $v \in\{0,1\}$.

Finally, we let $A$ and $B$ be the maps from $\left\{x_{1}, \ldots, x_{p-1}, y_{1}, \ldots, y_{p-1}, z\right\}$ to $E$ given (with exponential notation) by

$$
\begin{array}{ll}
z^{A}=z, & z^{B}=z, \\
x_{i}^{A}=x_{i+1}, & x_{i}^{B}=x_{p-i}, \quad \text { for each } i \in\{1, \ldots, p-2\}, \\
x_{p-1}^{A}=x_{1} \cdots x_{p-1}, & x_{p-1}^{B}=x_{1}, \\
y_{i}^{A}=y_{1} y_{i+1}, & y_{i}^{B}=y_{p-i}, \quad \text { for each } i \in\{1, \ldots, p-2\}, \\
y_{p-1}^{A}=y_{1}, & y_{p-1}^{B}=y_{1} .
\end{array}
$$

Lemma 2.2. Let $E, A$ and $B$ be as in Notation 2.1. Then $A$ and $B$ extend to two automorphisms $a$ and $b$ (respectively) of $E$. Moreover, $a^{p}=b^{2}=$ $(a b)^{2}=1$ and $\langle a, b\rangle$ is a dihedral group of order $2 p$.

Proof. To show that $A$ and $B$ extend to two automorphisms, say $a$ and $b$ respectively, of $E$ it suffices to prove that they preserve the defining relations of $E$. For instance, for every $i \in\{1, \ldots, p-2\}$, we have $\left[x_{i}^{A}, y_{i}^{A}\right]=$ $\left[x_{i+1}, y_{1} y_{i+1}\right]=\left[x_{i+1}, y_{i+1}\right]=z=z^{A}=\left[x_{i}, y_{i}\right]^{A}$. Similarly, $\left[x_{p-1}^{A}, y_{p-1}^{A}\right]=$ $\left[x_{1} \cdots x_{p-1}, y_{1}\right]=\left[x_{1}, y_{1}\right]=z=z^{A}=\left[x_{p-1}, y_{p-1}\right]^{A}$. For $i, j \in\{1, \ldots, p-2\}$ with $i \neq j$, we have $\left[x_{i}^{A}, y_{j}^{A}\right]=\left[x_{i+1}, y_{1} y_{j+1}\right]=1=\left[x_{i}, x_{j}\right]^{A}$. Moreover, $\left[x_{i}^{A}, y_{p-1}^{A}\right]=\left[x_{i+1}, y_{1}\right]=1=\left[x_{i}, y_{p-1}\right]^{A}$ and $\left[x_{p-1}^{A}, y_{j}^{A}\right]=\left[x_{1} \cdots x_{p-1}, y_{1} y_{j+1}\right]=$ $\left[x_{1}, y_{1}\right]\left[x_{j+1}, y_{j+1}\right]=z \cdot z=1=\left[x_{p-1}, y_{j}\right]^{A}$. All the other computations are similar and are left to the conscientious reader.

It is readily seen that $b^{2}$ fixes each generator $x_{1}, \ldots, x_{p-1}, y_{1}, \ldots, y_{p-1}, z$ of $E$, and hence $b^{2}=1$. Observe that $x_{p-1}^{a^{2}}=\left(x_{1} \cdots x_{p-2} x_{p-1}\right)^{a}=$ $x_{1}^{a} \cdots x_{p-2}^{a} x_{p-1}^{a}=x_{2} \cdots x_{p-1}\left(x_{1} \cdots x_{p-1}\right)=x_{1}$. ${ }^{i-1}$ Thus $x_{1}^{a^{p}}=\left(x_{1}^{a^{p-2}}\right)^{a^{2}}=$ $x_{p-1}^{a^{2}}=x_{1}$, and hence $x_{i}^{a^{p}}=\left(x_{1}^{a^{i-1}}\right)^{a^{p}}=\left(x_{1}^{a^{p}}\right)^{a^{i-1}}=x_{1}^{a^{i-1}}=x_{i}$ for every $i \in\{1, \ldots, p-1\}$. Arguing inductively on $i$, we get $y_{i}^{a^{p-1-i}}=y_{p-1-i} y_{p-1}$ for each $i \in\{1, \ldots, p-1\}$. It follows that $y_{i}^{a^{p-i}}=\left(y_{p-i-1} y_{p-1}\right)^{a}=y_{1} y_{p-i} y_{1}=$ $y_{p-i}$. Given $i \in\{1, \ldots, p-1\}$, applying this equality first to the index $i$ and then to the index $p-i$, we get $y_{i}^{a^{p}}=\left(y_{i}^{a^{p-i}}\right)^{a^{i}}=y_{p-i}^{a^{i}}=y_{p-(p-i)}=y_{i}$. Therefore $a^{p}$ fixes $x_{1}, \ldots, x_{p-1}, y_{1}, \ldots, y_{p-1}, z$ and hence $a^{p}=1$. Finally, we have

$$
\begin{array}{ll}
z^{a b}=z, & \\
x_{i}^{a b}=x_{i+1}^{b}=x_{p-i-1}, & \forall i \in\{1, \ldots, p-2\}, \\
x_{p-1}^{a b}=\left(x_{1} \cdots x_{p-1}\right)^{b}=x_{p-1} \cdots x_{1}=x_{1} \ldots x_{p-1}, & \\
y_{i}^{a b}=\left(y_{1} y_{i+1}\right)^{b}=y_{p-1} y_{p-i-1}, & \forall i \in\{1, \ldots, p-2\}, \\
y_{p-1}^{a b}=y_{1}^{b}=y_{p-1}, &
\end{array}
$$

from which it easily follows that $(a b)^{2}=1$. 
Proof of Theorem 1.1. Let $p, E, A$ and $B$ be as in Notation 2.1, let $a$ and $b$ be the automorphisms extending $A$ and $B$ as in Lemma 2.2, and set $M=\langle a, b\rangle$. Given two elements $n_{1}$ and $n_{2}$ of order $p$, define $N=\left\langle n_{1}\right\rangle \times\left\langle n_{2}\right\rangle \times E$ and $G=N \times M$ where

$$
n_{1}^{a}=n_{1}, n_{2}^{a}=n_{1} n_{2}, n_{1}^{b}=n_{1}, n_{2}^{b}=n_{2}^{-1}
$$

(note that this is well-defined because the action of $M=\langle a, b\rangle$ on $\left\langle n_{1}, n_{2}\right\rangle$ determines a dihedral group of automorphisms of $\left\langle n_{1}, n_{2}\right\rangle$ order $2 p$ ). We show that $B(G)=K_{2.5}$.

We start by determining the conjugacy class sizes of $G \backslash \mathbf{Z}(G)$. Clearly, $\mathbf{Z}(G)=\left\langle n_{1}, z\right\rangle$.

We claim that the conjugacy classes of $G$ in $N \backslash \mathbf{Z}(G)$ have cardinality $2 p$ or $4 p$. Let $e \in\{h \in E \mid[a, h] \in \mathbf{Z}(E)\}$ and write $e=x_{1}^{\varepsilon_{1}} \cdots x_{p-1}^{\varepsilon_{p-1}} y_{1}^{\eta_{1}} \cdots y_{p-1}^{\eta_{p-1}} z^{\nu}$, for some $\varepsilon_{1}, \ldots, \varepsilon_{p-1}, \eta_{1}, \ldots, \eta_{p-1}, v \in\{0,1\}$. We have

$$
\begin{aligned}
e^{a} & =\left(x_{1}^{\varepsilon_{1}}\right)^{a} \cdots\left(x_{p-1}^{\varepsilon_{p-1}}\right)^{a}\left(y_{1}^{\eta_{1}}\right)^{a} \cdots\left(y_{p-1}^{\eta_{p-1}}\right)^{a}\left(z^{v}\right)^{a} \\
& =x_{2}^{\varepsilon_{1}} x_{3}^{\varepsilon_{2}} \cdots x_{p-1}^{\varepsilon_{p-2}}\left(x_{1} \cdots x_{p-1}\right)^{\varepsilon_{p-1}}\left(y_{1} y_{2}\right)^{\eta_{1}}\left(y_{1} y_{3}\right)^{\eta_{2}} \cdots\left(y_{1} y_{p-1}\right)^{\eta_{p-2}} y_{1}^{\eta_{p-1}} z^{v} \\
& =x_{1}^{\varepsilon_{p-1}} x_{2}^{\varepsilon_{1}+\varepsilon_{p-1}} \cdots x_{p-1}^{\varepsilon_{p-2}+\varepsilon_{p-1}} y_{1}^{\eta_{1}+\cdots+\eta_{p-1}} y_{2}^{\eta_{1}} \cdots y_{p-1}^{\eta_{p-2}} z^{v} .
\end{aligned}
$$

As $e^{-1} e^{a}=[e, a] \in \mathbf{Z}(E)=\langle z\rangle$, by comparing $e$ with $e^{a}$, we get $\varepsilon_{i}=$ $\eta_{i}=0$ for every $i \in\{1, \ldots, p-1\}$, and hence $e \in \mathbf{Z}(E)$. Thus $\{h \in E \mid[a, h] \in \mathbf{Z}(E)\}=\mathbf{Z}(E)$. In particular, $\mathbf{C}_{E}(a)=\mathbf{Z}(E)$ and $a$ acts fixed point freely on $E \backslash \mathbf{Z}(E)$. It follows that the orbits of $M$ acting by conjugation on $E \backslash \mathbf{Z}(E)$ have size $p$ or $2 p$. Since $\left|E^{\prime}\right|=2$, for every $e \in E \backslash \mathbf{Z}(E)$ we have $\left|E: \mathbf{C}_{E}(e)\right|=2$. Thus $e^{G}$ has size $2 p$ or $4 p$. Now let $g \in N \backslash \mathbf{Z}(N)$. Write $g=n e$, for some $n \in\left\langle n_{1}, n_{2}\right\rangle \backslash\left\langle n_{1}\right\rangle$ and $e \in E \backslash\langle z\rangle$. Now $\mathbf{C}_{N}(g)=$ $\left\langle n_{1}, n_{2}\right\rangle \mathbf{C}_{E}(e)$ has index 2 in $N$ and so (as $a$ does not centralise $n$ ) $\mathbf{C}_{G}(g)$ has index divisible by $2 p$. It follows (with an elementary computation) that the conjugacy classes of $G$ in $N \backslash \mathbf{Z}(G)$ have size $2 p$ or $4 p$. Observe that both possibilities can occur: $\left|x_{1}^{G}\right|=4 p$ and $\left|\left(x_{1} x_{p-1}\right)^{G}\right|=2 p$.

Let $g \in\langle N, a\rangle \backslash N$ and write $g=n a^{i}$, for some $n \in N$ and $i \in$ $\{1, \ldots, p-1\}$. Now, given $e \in E$, we have $e^{n}=e z^{v}$, for some $v \in\{0,1\}$. In particular, if $e \in \mathbf{C}_{E}(g)$, then $e=e^{g}=\left(e^{n}\right)^{a^{i}}=\left(e z^{v}\right)^{a^{i}}=e^{a^{i}} z^{v}$, and hence $[a, e] \in \mathbf{Z}(E)$. Thus $e \in \mathbf{Z}(E)$. It follows that $\mathbf{C}_{N}(g)=\mathbf{Z}(G)$ and $\left|\mathbf{C}_{G}(g)\right|=$ $p\left|\mathbf{C}_{N}(g)\right|=2 p^{2}$. Thus $\left|g^{G}\right|=2^{2 p-1} p$.

It remains to compute the size of the conjugacy classes of $G$ in $G \backslash\langle a, N\rangle$. Observe that every element of $G \backslash\langle a, N\rangle$ is conjugate to an element of $\langle b, N\rangle \backslash N$. In particular, since we are interested only on the conjugacy class sizes, we may assume that $g \in\langle b, N\rangle \backslash N$. Write $g=n b$, for 
some $n \in N$. Observe that

$$
\begin{aligned}
\{h \in E \mid[h, b] \in \mathbf{Z}(E)\}= & \left\langle x_{1} x_{p-1}, x_{2} x_{p-2}, \ldots, x_{(p-1) / 2} x_{(p+1) / 2},\right. \\
& \left.y_{1} y_{p-1}, y_{2} y_{p-2}, \ldots, y_{(p-1) / 2} y_{(p+1) / 2}, z\right\rangle=\mathbf{C}_{E}(b),
\end{aligned}
$$

and $\left|\mathbf{C}_{E}(b)\right|=2^{p}$. Now, arguing as above, it is easy to verify that $\mathbf{C}_{N}(g) \leq\left\langle n_{1}, \mathbf{C}_{E}(b)\right\rangle$ and $\left|\left\langle n_{1}, \mathbf{C}_{E}(b)\right\rangle: \mathbf{C}_{N}(g)\right| \in\{1,2\}$. Thus $\left|\mathbf{C}_{G}(g)\right| \in$ $\left\{p^{2} 2^{p}, p^{2} 2^{p+1}\right\}$ and hence $\left|g^{G}\right|$ equals $2^{p-1} p^{2}$ or $2^{p} p^{2}$. Observe that both of these cases can occur: in fact $\left|b^{G}\right|=\left|G: \mathbf{C}_{G}(b)\right|=2^{p-1} p^{2}$ and $\left|\left(x_{1} b\right)^{G}\right|=$ $\left|G: \mathbf{C}_{G}\left(x_{1} b\right)\right|=2^{p} p^{2}$.

Summing up, the conjugacy class sizes $\left|g^{G}\right|$ (as $g$ runs through $G \backslash \mathbf{Z}(G)$ ) are $2 p, 4 p, 2^{2 p-1} p, 2^{p-1} p^{2}, 2^{p} p^{2}$, from which it follows that $B(G)=K_{2,5}$.

\section{The case $B(G)=K_{2,4}$}

Here we describe a group $G$ with $B(G)=K_{2,4}$ : the construction is due to Casolo [7]. Let $Q$ be the quaternion group of order 8, and let $Q_{1}=\left\langle q_{1}\right\rangle$, $Q_{2}=\left\langle q_{2}\right\rangle$ and $Q_{3}=\left\langle q_{3}\right\rangle$ be the three maximal subgroups of $Q$. Let $p$ be an odd prime and let $E$ be the extra-special $p$-group

$$
\begin{aligned}
\left\langle x_{1}, x_{2}, x_{3}, y_{1}, y_{2}, y_{3}, z\right| x_{i}^{p}=y_{i}^{p}=z^{p}=\left[x_{i}, z\right]=\left[y_{i}, z\right]=1 \forall i, \\
{\left.\left[x_{i}, x_{j}\right]=\left[y_{i}, y_{j}\right]=\left[x_{i}, y_{j}\right]=1 \forall i \neq j,\left[x_{i}, y_{i}\right]=z \forall i\right\rangle . }
\end{aligned}
$$

Arguing as in Section 2, it is easy to verify that by assigning

$x_{1}^{q_{1}}=x_{1}, \quad y_{1}^{q_{1}}=y_{1}, \quad x_{2}^{q_{1}}=x_{2}^{-1}, \quad y_{2}^{q_{1}}=y_{2}^{-1}, \quad x_{3}^{q_{1}}=x_{3}^{-1}, \quad y_{3}^{q_{1}}=y_{3}^{-1}, \quad z^{q_{1}}=z$,

$x_{1}^{q_{2}}=x_{1}^{-1}, \quad y_{1}^{q_{2}}=y_{1}^{-1}, \quad x_{2}^{q_{2}}=x_{2}, \quad y_{2}^{q_{2}}=y_{2}, \quad x_{3}^{q_{2}}=x_{3}^{-1}, \quad y_{3}^{q_{2}}=y_{3}^{-1}, \quad z^{q_{2}}=z$,

$x_{1}^{q_{3}}=x_{1}^{-1}, \quad y_{1}^{q_{3}}=y_{1}^{-1}, \quad x_{2}^{q_{3}}=x_{2}^{-1}, \quad y_{2}^{q_{3}}=y_{2}^{-1}, \quad x_{3}^{q_{3}}=x_{3}, \quad y_{3}^{q_{3}}=y_{3}, \quad z^{q_{3}}=z$,

we define a group-action of $Q$ on $E$. Set $G=E \times Q$.

Now, following the proof of Theorem 1.1 we see that the conjugacy class sizes $\left|g^{G}\right|$, as $g$ runs through $G \backslash \mathbf{Z}(G)$, are $2 p, 4 p, 2 p^{4}$ and $2 p^{5}$. In particular, $B(G)=K_{2,4}$.

\section{REFERENCES}

[1] G. Alfandary, On graphs related to conjugacy classes of groups, Israel J. Math. 86 (1994), 211-220.

[2] A. Azad, M. A. Iranmanesh, C. E. Praeger, P. Spiga, Abelian coverings of finite general linear groups and an application to their non-commuting graphs, J. Algebraic Combin. 34 (2011), 683-711. 
[3] E. A. Bertram, M. Herzog, A. Mann, On a graph related to conjugacy classes of groups, Bull. London Math. Soc. 22 (1990), 569-575.

[4] D. Bubboloni, S. Dolfi, M. A. Iranmanesh, C. E. Praeger, On bipartite divisor graphs for group conjugacy class sizes, J. Pure Appl. Algebra 213 (2009), 1722-1734.

[5] A. R. Camina, R. D. Camina, The influence of conjugacy class sizes on the structure of finite groups: a survey, Asian-European J. Math. 4 (2011), 559588.

[6] C. Casolo, S. Dolfi, The diameter of a conjugacy class graph of finite groups, Bull. London Math. Soc. 28 (1996), 141-148.

[7] C. Casolo, personal communication.

[8] S. Dolfi, E. JaBARA, The structure of finite groups of conjugate rank 2, Bull. London Math. Soc. 41 (2009), 916-926.

[9] S. Dolfi, E. Pacifici, L. SAnus, P. SPIGa, On the vanishing prime graph of groups, Journal Lond. Math. Soc. 82 (2010), 167-183.

[10] R. Hafezieh, M. A. Iranmanesh, Bipartite divisor graph for the product of subsets of integers, Bull. Aust. Math. Soc. 87 (2013), 288-297.

[11] R. HAFEZIEH, P. SPIGA, Groups having complete bipartite divisor graphs for their conjugacy class sizes, arXiv:1309.5629.

[12] M. A. Iranmanesh, C. E. Praeger, Bipartite divisor graphs for integer subsets, Graphs Combin. 26 (2010), 95-105.

[13] L. S. KaZARIN, On groups with isolated conjugacy classes, Izv. Vyssh. Uchebn. Zaved. Mat. 25 (1981), 40-45.

[14] M. L. LEWIS, An overview of graphs associated with character degrees and conjugacy class sizes in finite groups, Rocky Mountain J. Math. 38 (2008), $175-211$.

[15] B. TAERI, Cycles and bipartite graph on conjugacy class of groups, Rend. Semin. Mat. Univ. Padova 123 (2010), 233-247.

[16] J. S. Williams, Prime graph components of finite groups, J. Algebra 69 (1981), 487-513.

Manoscritto pervenuto in redazione il 22 settembre 2013. 
